\title{
Collisional energy transfer in two-component plasmas
}

\author{
J. Vranjes, ${ }^{1,2}$ M. Kono, ${ }^{3}$ S. Poedts, ${ }^{1}$ and M. Y. Tanaka ${ }^{4}$ \\ ${ }^{1}$ Center for Plasma Astrophysics and Leuven Mathematical Modeling and Computational Science Center, \\ Celestijnenlaan 200B, 3001 Leuven, Belgium \\ ${ }_{3}^{2}$ Faculté des Sciences Appliquées, Avenue F. D. Roosevelt 50, 1050 Bruxelles, Belgium \\ ${ }^{3}$ Faculty of Policy Studies, Chuo University, Hachioji, Tokyo 192-0393, Japan \\ ${ }^{4}$ Department of High Energy Engineering Science, Kyushu University, Kasuga-koen 6-1, Kasuga, Fukuoka \\ 816-8580, Japan
}

(Received 14 July 2008; accepted 14 August 2008; published online 11 September 2008)

The friction in plasmas consisting of two species with different temperatures is discussed together with the consequent energy transfer. It is shown that the friction between the two species has no effect on the ion acoustic mode in a quasineutral plasma. Using the Poisson equation instead of the quasineutrality reveals the possibility for an instability driven by the collisional energy transfer. However, the different starting temperatures of the two species imply an evolving background. It is shown that the relaxation time of the background electron-ion plasma is, in fact, always shorter than the growth rate time. Therefore the instability is unlikely to develop. The results obtained here should contribute to the definite clarification of some contradictory results obtained in the past. (C) 2008 American Institute of Physics. [DOI: 10.1063/1.2977987]

\section{INTRODUCTION}

Plasmas both in the laboratory and in space are frequently in the state of partial thermodynamic equilibrium ${ }^{1-3}$ (i.e., with an initial temperature disparity of the plasma constituents). Collisions in such plasmas will after some time eventually result in equal temperatures of the species, implying an evolving plasma. There exists a longstanding controversy in the literature which deals with the effects of this temperature disparity on the perturbations in such an evolving plasma, more precisely, on the ion acoustic (IA) waves.

In Ref. 4, it is claimed that the corresponding energy transfer may result in the instability of the acoustic mode at large wavelengths (within the quasineutrality limit), and that this growth may be described within the fluid theory. The necessary condition for the instability obtained in Ref. 4 for an electron-ion plasma is, in fact, very easily satisfied because it requires only a very small temperature difference between the two species (electrons and ions); viz., $T_{e}$ $>4 T_{i} / 3$. This instability condition is obtained by using the energy equations including the source/sink terms originating from the collisional transfer, together with the corresponding friction force terms in the momentum equations. The sufficient instability condition is stronger because of additional dissipative effects, such as viscosity and thermal conductivity.

However, the currentless instability described in Ref. 4 is based on a model which disregards the same temperature disparity in the description of the spatially homogeneous background, which, for the same reasons, must be time evolving. In other words, the effects of collisions in the background plasma have been explicitly neglected. Note that here and further in the text, because of the time evolution, the term "background" is used instead of the equilibrium. These effects of collisions have been discussed in Ref. 5, published one year after Ref. 4, and for the same quasineutrality case. There, it is claimed that there is no instability for any tem- perature ratio of the two plasma components, and, moreover, that this holds even in a current-carrying plasma, as long as the difference between the electron and ion equilibrium velocity remains below the sound speed. All that was needed to come to that conclusion was to let the background plasma evolve freely in the presence of the given temperature difference. However, we observe that Ref. 5 has apparently remained almost unnoticed by researchers, in contrast to the widely cited Ref. 4 (see, e.g., Refs. 6-10 and many others).

In the present work, this controversy is revisited for any two-component plasma. Essential for the problem is the energy equation describing the temperature variation. In the simplified form that we shall use, it contains only the collisional energy transfer source/sink term on the right-hand side. This simplified form is used for clarity only because, according to Ref. 4 , in the absence of currents, that term alone is supposed to yield an instability. In view of the controversy mentioned above, here we give some details following Braginskii, ${ }^{11}$ where the energy equation for any species $a$ is given in the form

$$
\frac{3}{2}\left(\frac{\partial}{\partial t}+\vec{v}_{a} \cdot \nabla\right) n_{a} T_{a}+\frac{5}{2} n_{a} T_{a} \nabla \cdot \vec{v}_{a}=Q_{a} .
$$

The corresponding equation for the species $b$ has the same shape, but with a minus sign on the right-hand side. We use the Landau formula for the energy transfer source/sink term, ${ }^{12} Q_{a}=3 m_{b} \nu_{b a} n_{b}\left(T_{b}-T_{a}\right) / m_{a}$, where ${ }^{13}$

$$
\nu_{b a}=4\left(\frac{2 \pi}{m_{b}}\right)^{1 / 2}\left(\frac{q_{a} q_{b}}{4 \pi \varepsilon_{0}}\right)^{2} \frac{n_{a} L_{b a}}{3\left(T_{b}+T_{a} m_{b} / m_{a}\right)^{3 / 2}} .
$$

The Coulomb logarithm is given by $L_{b a}=\ln \left[r_{d} / b_{0}\right]$, $r_{d}=r_{d a} r_{d b} /\left(r_{d a}^{2}+r_{d b}^{2}\right)^{1 / 2}, \quad r_{d j}=v_{T j} / \omega_{p j}, \quad$ and $\quad b_{0}$ $=\left[\left|q_{a} q_{b}\right| /\left(4 \pi \varepsilon_{0}\right)\right] /\left[3\left(T_{a}+T_{b}\right)\right]$ is the impact parameter. With the use of the continuity, Eq. (1) is transformed into 


$$
\frac{3}{2} n_{a} \frac{\partial T_{a}}{\partial t}+n_{a} T_{a} \nabla \cdot \vec{v}_{a}+\frac{3}{2} n_{a}\left(\vec{v}_{a} \cdot \nabla\right) T_{a}=Q_{a} .
$$

The additional source/sink term of the form $\vec{F}_{f a} \cdot\left(\vec{v}_{a}-\vec{v}_{b}\right)$, where $\vec{F}_{f a}$ is the friction force acting on the species $a$, in the absence of equilibrium currents/drifts, is in fact nonlinear and will not be discussed here. The other (sink) terms, due to viscosity and thermal conductivity, are omitted only for the sake of clarity; i.e., in order to demonstrate more clearly the effect of the disputed collisional energy transfer term. The effect of these omitted terms is easily predictable. Equation (3) is valid for any species $a, b$, thus including the electronion plasma from Refs. 4-9.

\section{MODEL WITH A NONEVOLVING BACKGROUND}

In Ref. 4 the collisions in the equilibrium were explicitly ignored. In that case, the two perturbed energy equations without the evolving background effects, corresponding to the model from Ref. 4 read

$$
\begin{aligned}
& \frac{\partial T_{(a, b) 1}}{\partial t}+\frac{2}{3} T_{(a, b) 0} \nabla \cdot \vec{v}_{(a, b) 1} \\
& = \pm 2 \frac{m_{b}}{m_{a}} \nu_{b a}\left(T_{b 1}-T_{a 1}\right) \pm 2 \nu_{b a} \frac{m_{b}}{m_{a}}\left(T_{b 0}-T_{a 0}\right) \frac{n_{b 1}}{n_{0}} .
\end{aligned}
$$

Here, the minus sign applies to the species $b$.

The momentum equation which we use throughout the text for the species $a$ is of the form $m_{a} n_{a} \partial \vec{v}_{a} / \partial t=-q_{a} n_{a} \nabla \phi$ $-\nabla\left(n_{a} T_{a}\right)-m_{a} n_{a} \nu_{a b}\left(\vec{v}_{a}-\vec{v}_{b}\right)$, and the continuity equation has its standard form. Similar equations are used for the species $b$, where the friction term is of the form $\vec{F}_{f b}=-m_{b} n_{b} \nu_{b a}\left(\vec{v}_{b}\right.$ $-\vec{v}_{a}$ ).

In the case of quasineutral perturbations, the two number densities $n_{(a, b) 1}$ are calculated from the continuity equations and are made equal, as in Refs. 4 and 5 (this is typically valid when dealing with wavelengths that are much longer than the Debye length). The dispersion equation in that case reads

$$
\left(\omega+\frac{i 4 m_{b} \nu_{b a}}{m_{a}}\right)\left(\omega^{2}-\frac{5 k^{2}}{3} \frac{T_{a 0}+T_{b 0}}{m_{a}+m_{b}}\right)=0 .
$$

Hence, even using the same model as in Ref. 4, we conclude that there is neither an instability nor damping of the acoustic mode, regardless of the ratio $T_{a 0} / T_{b 0}$.

Note that the momentum conservation condition $\nu_{a b}$ $=m_{b} n_{b} \nu_{b a} /\left(m_{a} n_{a}\right)$ is nowhere used in the derivation of Eq. (5). This is because the friction terms vanish in any case. In fact, from the two continuity equations we have the velocities $v_{a 1}-v_{b 1}=(\omega / k)\left(n_{a 1} / n_{a 0}-n_{b 1} / n_{b 0}\right)$. Using $q_{a} n_{a}=q_{b} n_{b}$ and assuming the constant charge on the two species $a$ and $b$, we have $v_{a 1}-v_{b 1}=0$, so that the assumption of quasineutrality cancels the friction completely. Otherwise, for a varying charge we would have $v_{a 1}-v_{b 1}=\left[\omega n_{a 1} /\left(k n_{a 0}\right)\right][1$ $\left.-z_{a 1} z_{b 0} /\left(z_{b 1} z_{a 0}\right)\right]$, where $z_{j}$ denotes the charge number, and therefore the friction effects would remain. Further in the text we assume singly charged species.

\section{ISOTHERMAL CASE}

\section{A. Quasineutral perturbations}

The derivations are now repeated for isothermal quasineutral perturbations. In addition, the energy equation may be omitted in the equilibrium also assuming that the relaxation time for the equilibrium temperature is much longer than the period of wave oscillations. Keeping the full friction force $\vec{F}_{f}$ in both momentum equations, and within the same quasineutrality limit, yields a real dispersion equation $\omega^{2}=k^{2}\left(T_{a 0}+T_{b 0}\right) /\left(m_{a}+m_{b}\right)$. Hence, in the given limit the collisions (through friction) do not affect the isothermal ion acoustic mode.

This fact is usually overlooked in the literature where, in the limit $m_{a} \gg m_{b}$, a typical mistake is that the friction is kept for the lighter species only, in the incomplete simplified form $\vec{F}_{f b}=-m_{b} n_{b} \nu_{b a} \vec{v}_{b}$. For an electron-ion plasma this gives the incorrect result $\omega= \pm k\left(c_{s}^{2}+v_{T i}^{2}\right)^{1 / 2}-\nu_{e i} / 2$. We stress again that the collisions appear in Eq. (5) only from the energy equations, yet they do not affect the IA mode.

\section{B. Non-quasineutral perturbations}

Using the Poisson equation instead of quasineutrality, for isothermal perturbations we obtain coupled and damped IA and Langmuir waves

$$
\begin{aligned}
\omega^{4}+ & i\left(\nu_{a b}+\nu_{b a}\right) \omega^{3}-\left[k^{2}\left(v_{T a}^{2}+v_{T b}^{2}\right)+\omega_{p a}^{2}+\omega_{p b}^{2}\right] \omega^{2} \\
& -i k^{2}\left(\nu_{a b} v_{T b}^{2}+\nu_{b a} v_{T a}^{2}\right) \omega+k^{4} v_{T a}^{2} v_{T b}^{2} \\
& +k^{2}\left(v_{T a}^{2} \omega_{p b}^{2}+v_{T b}^{2} \omega_{p a}^{2}\right)=0 .
\end{aligned}
$$

In the collisionless limit, the two modes [Eq. (6)] decouple by setting $T_{a}=T_{b}$, though in some situations this may make no sense because in this case the acoustic mode may lose its electrostatic nature, especially in pair-plasmas. For a pair (pair-ion, electron-positron) collisionless plasma the solutions are ${ }^{14} \omega^{2}=\omega_{p}^{2}+k^{2}\left(v_{T a}^{2}+v_{T b}^{2}\right) / 2 \pm\left[\omega_{p}^{4}+k^{4}\left(v_{T a}^{2}-v_{T b}^{2}\right) / 4\right]^{1 / 2}$.

In the low frequency limit $\omega \ll \omega_{p(a, b)}$ and for an electron-ion plasma, from Eq. (6) we have $\omega^{2}=k^{2} v_{s}^{2}$ $-i 2 \nu_{e i} \omega m_{e} r_{d e}^{2} k^{2} / m_{i}$, so that the IA mode is damped:

$$
\omega= \pm k v_{s}\left(1-r_{d e}^{2} k^{2} \frac{\nu_{i e}^{2} r_{d e}^{2}}{v_{s}^{2}}\right)^{1 / 2}-i \nu_{i e} r_{d e}^{2} k^{2}
$$

Here, $v_{s}^{2}=c_{s}^{2}+v_{T i}^{2}$, and we have used the momentum conservation $\nu_{i e}=m_{e} \nu_{e i} / m_{i}$. Note that the damping in Eq. (7) is $k$-dependent.

\section{EVOLVING BACKGROUND PLASMA}

From Eq. (3) it is seen that in a quasineutral homogeneous background, without flows/currents, the background temperature is also evolving in time as

$$
\frac{\partial T_{(a, b) 0}}{\partial t}= \pm 2 \frac{m_{b}}{m_{a}} \nu_{b a}\left(T_{b 0}-T_{a 0}\right) .
$$

Keeping the collision frequencies constant (the approximation discussed below), this gives the temperatures for the two species 


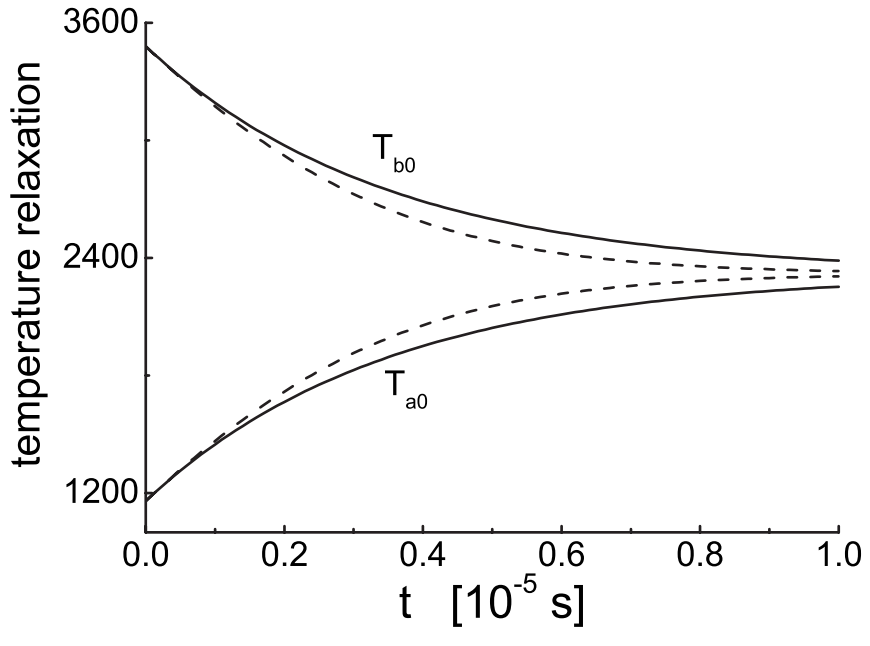

FIG. 1. Approximative (full lines) and exact relaxation with time-dependent collision frequencies (dashed lines) of the background plasma temperatures [Eq. (8)].

$$
\begin{aligned}
T_{(a, b) 0}(t)= & \left\{\hat{T}_{(a, b) 0}\left[1+\exp \left(-4 \nu_{a b} t\right)\right]\right. \\
& \left.+\hat{T}_{(a, b) 0}\left[1-\exp \left(-4 \nu_{a b} t\right)\right]\right\} / 2 .
\end{aligned}
$$

It is seen that they evolve towards the common value $\left(\hat{T}_{a 0}\right.$ $\left.+\hat{T}_{b 0}\right) / 2$.

On the other hand, solving Eq. (8) numerically, with time-dependent collision frequencies [Eq. (2)] gives a slightly faster relaxation for the two temperatures. To get a feeling on the relaxation time scale, this is presented in Fig. 1 by taking $n_{0}=10^{18} \mathrm{~m}^{-3}$ and $\hat{T}_{a 0}=0.1 \mathrm{eV}, \hat{T}_{b 0}=3 \hat{T}_{a 0}$.

Equation (8) is to be used in the linearization of Eq. (3), which in the case $n_{a 0}=n_{b 0}=n_{0}$ yields

$$
\begin{aligned}
\frac{\partial T_{a 1}}{\partial t}+\frac{2}{3} T_{a 0} \nabla \cdot \vec{v}_{a 1}= & +2 \frac{m_{b}}{m_{a}} \nu_{b a}\left(T_{b 1}-T_{a 1}\right) \\
& -2 \nu_{b a} \frac{m_{b}}{m_{a}} \frac{n_{a 1}-n_{b 1}}{n_{0}}\left(T_{b 0}-T_{a 0}\right) .
\end{aligned}
$$

The corresponding equation for the component $b$ is

$$
\frac{\partial T_{b 1}}{\partial t}+\frac{2}{3} T_{b 0} \nabla \cdot \vec{v}_{b 1}=-2 \frac{m_{b}}{m_{a}} \nu_{b a}\left(T_{b 1}-T_{a 1}\right) .
$$

Here, in the process of linearization yielding Eq. (10), the term $(3 / 2) n_{b 1} \partial T_{b 0} / \partial t$ on the left-hand side, cancels out with the term $-\left(m_{b} / m_{a}\right) \nu_{b a}\left(T_{b 0}-T_{a 0}\right) n_{b 1}$ on the right-hand side after using the background equation (8) for the species $b$.

Hence, both Eqs. (9) and (10) are obtained taking into account the evolution of the background. There appears an additional asymmetry between the two energy equations (apart from the opposite signs of the first term on the righthand side), due to the last term in Eq. (9). This extra asymmetry is a consequence of the fact that the internal energy of the two species may also change due to the presence of the new ingredient in the system, i.e., the perturbed electric field $n_{a 1}-n_{b 1}=\varepsilon_{0} \nabla \cdot \vec{E}_{1} / e$ (in the presence of the necessary collisions, of course). However, it vanishes if the quasineutrality condition is used on the right-hand side in Eq. (9). This may sometimes be permissible in higher order terms but not in general, for example assuming that the source/sink term in the energy equations gives only small imaginary corrections to the frequency.

However, regardless of the fact that the last term in Eq. (9) is used or not, the effects of the evolving background remain within Eq. (9) in both cases. Note also that the cancellation of the terms in the equation for the species $b$ (which is due to evolving background as described above) remains intact regardless if the quasineutrality is used or not.

\section{A. Quasineutral case}

We stress that Eq. (5) is obtained also by using Eqs. (9) and (10) in the quasineutral limit [implying that the last term in Eq. (9) is omitted]. Hence, the IA mode appears unaffected by friction in the quasineutral limit even if the energy equations are used, and if the background is described correctly as evolving.

\section{B. Non-quasineutral case}

We now use the two energy equations (9) and (10) with the Poisson equation. The dispersion equation becomes

$$
\begin{aligned}
\omega^{6}+ & i \nu_{b a}\left(1+\frac{5 m_{b}}{m_{a}}\right) \omega^{5}-\omega^{4}\left[\frac{5}{3} k^{2}\left(v_{T a}^{2}+v_{T b}^{2}\right)+\omega_{p a}^{2}+\omega_{p b}^{2}+4 v_{b a}^{2} \frac{m_{b}}{m_{a}}\left(1+\frac{m_{b}}{m_{a}}\right)\right]+i \omega^{3} \nu_{b a}\left[k^{2} v_{T b}^{2} \frac{2 m_{b}^{2}}{m_{a}^{2}}-k^{2} v_{T a}^{2}\left(\frac{5}{3}+\frac{22 m_{b}}{3 m_{a}}\right)\right. \\
& \left.-4 \omega_{p a}^{2}\left(1+\frac{m_{b}}{m_{a}}\right)-7 k^{2} v_{T b}^{2} \frac{m_{b}}{m_{a}}\right]+\omega^{2}\left\{\frac{25}{9} k^{4} v_{T a}^{2} v_{T b}^{2}+\frac{5 k^{2}}{3}\left(v_{T a}^{2} \omega_{p b}^{2}+v_{T b}^{2} \omega_{p a}^{2}\right)+4 k^{2} \nu_{b a}^{2} \frac{m_{b}}{m_{a}}\left[v_{T a}^{2}\left(\frac{2}{3}+\frac{m_{b}}{m_{a}}\right)\right.\right. \\
& \left.\left.+v_{T b}^{2} \frac{m_{b}^{2}}{m_{a}^{2}}\left(\frac{8}{3}-\frac{m_{b}}{m_{a}}\right)\right]\right\}+i 10 \omega \nu_{b a} k^{2}\left[k^{2} v_{T b}^{2} \frac{m_{b}}{m_{a}}\left(v_{T a}^{2}-\frac{v_{T b}^{2}}{3} \frac{m_{b}}{m_{a}}\right)+\frac{2}{3} \omega_{p a}^{2}\left(v_{T a}^{2}+v_{T b}^{2} \frac{m_{b}}{m_{a}}\right)\right]+4 k^{4} \nu_{b a}^{2} \frac{m_{b}}{m_{a}}\left(v_{T a}^{2}-v_{T b}^{2} \frac{m_{b}}{m_{a}}\right)^{2}=0 .
\end{aligned}
$$

Solving for the IA mode yields approximately

$$
\omega_{\mathrm{IA}}^{2}=\frac{5 k^{2}\left(\omega_{p b}^{2} v_{T a}^{2}+\omega_{p a}^{2} v_{T b}^{2}+5 k^{2} v_{T a}^{2} v_{T b}^{2} / 3\right)}{3\left[\omega_{p a}^{2}+\omega_{p b}^{2}+(5 / 3) k^{2}\left(v_{T a}^{2}+v_{T b}^{2}\right)\right]} .
$$


The corresponding growth rate is

$$
\begin{aligned}
\gamma= & \frac{1}{2\left[\omega_{p a}^{2}+\omega_{p b}^{2}+(5 / 3) k^{2}\left(v_{T a}^{2}+v_{T b}^{2}\right)-2 \omega_{\mathrm{IA}}^{2}\right]}\left\{\nu_{a b}\left[\omega_{\mathrm{IA}}^{2}-(5 / 3) k^{2} v_{T b}^{2}\right]\left[1+(4 / 3) k^{2} v_{T a}^{2} / \omega_{\mathrm{IA}}^{2}\right]+\nu_{b a}\left[\omega_{\mathrm{IA}}^{2}-(5 / 3) k^{2} v_{T a}^{2}\right]\right. \\
& \times\left[1+(4 / 3) \nu_{a b} k^{2} v_{T b}^{2} /\left(\nu_{b a} \omega_{\mathrm{IA}}^{2}\right)\right]+2\left(1-T_{a 0} / T_{b 0}\right)\left(\nu_{a b} k^{2} c_{s}^{2} / \omega_{\mathrm{IA}}^{2}\right)\left[\omega_{\mathrm{IA}}^{2}-(5 / 3) k^{2} v_{T b}^{2}-\left(8 \nu_{a b} \nu_{b a} / \omega_{\mathrm{IA}}^{2}\right)\left(\omega_{\mathrm{IA}}^{2}-k^{2} v_{T a}^{2}\right)\right. \\
& \left.\left.+\left(8 \nu_{a b}^{2} / \omega_{\mathrm{IA}}^{2}\right)\left(\omega_{\mathrm{IA}}^{2}-k^{2} v_{T b}^{2}\right)\right]\right\} .
\end{aligned}
$$

In principle, Eq. (13) reveals the possibility for a growing IA mode if the Poisson equation is used instead of the quasineutrality in a time-evolving background plasma. For example, this can be easily demonstrated in the limit of negligible terms originating from the last term in Eq. (9). This is permissible on condition $\left|\left(T_{a 1}-T_{b 1}\right) /\left(n_{a 1}-n_{b 1}\right)\right| \gg\left|T_{a 0}-T_{b 0}\right| / n_{0}$, or in an alternative form, $\left|\left(T_{a 1}-T_{b 1}\right) /\left(T_{a 0}-T_{b 0}\right)\right|$ $\gg r_{d b}^{2} k^{2}\left|q_{b} \phi_{1}\right| / T_{b 0}$. In that limit, the numerical solution of Eq. (11) yields the growth rate of the IA mode in an electron-ion plasma that is presented in Fig. 2. Here, $n_{0}=10^{18} \mathrm{~m}^{-3}$, and we take several values of $T_{e} / T_{i}$, where $T_{i}=0.1 \mathrm{eV}$. The growth rate increases with $T_{e} / T_{i}$, but only up to $T_{e} / T_{i} \simeq 3$. For even higher values of the temperature ratio the instability ceases, this is represented by the dashed $\left(T_{e} / T_{i} \simeq 10\right)$ line.

However, we stress that the system evolves in time. Thus, in order to have a reasonably fast growth of the perturbations, the following condition must be satisfied [cf. Eq. (8)]:

$$
\gamma_{r} \equiv 2\left(m_{b} / m_{a}\right) \nu_{b a} \ll \gamma
$$

Here, $\gamma_{r}^{-1}$ determines the relaxation time for the background. Taking the electron-ion case as in Ref. 4 and the corresponding self-evident conditions $m_{b} \ll m_{a}, T_{a 0}<T_{b 0}, k^{2} v_{T a}^{2}<\omega_{\text {IA }}^{2}$ $<\omega_{p a}^{2}<\omega_{p b}^{2}$, from Eq. (13) to the leading order terms we

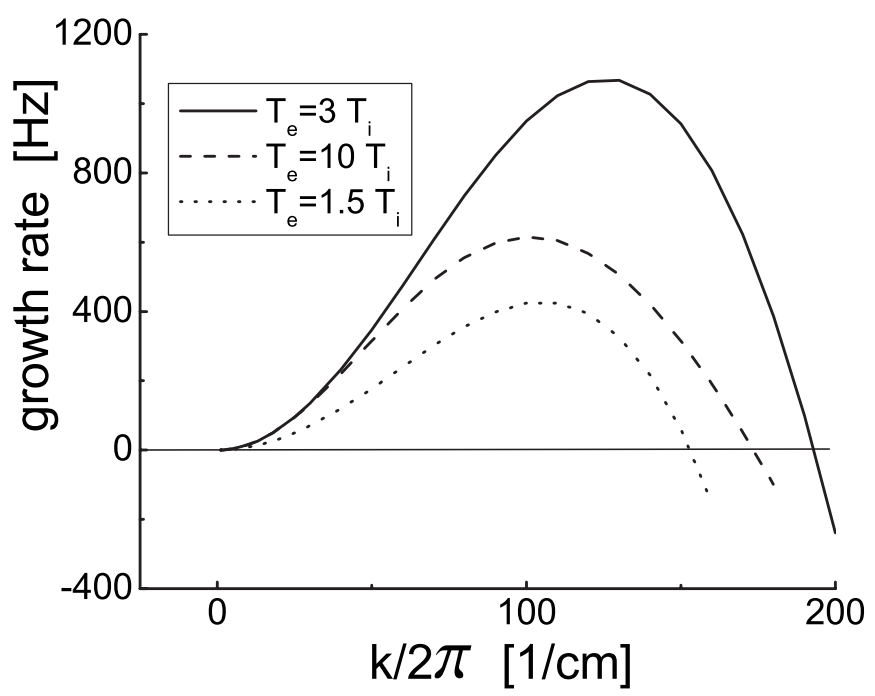

FIG. 2. The growth rate of the IA mode in electron-proton plasma with $n_{0}$ $=10^{18} \mathrm{~m}^{-3}$ and for several values of $T_{e} / T_{i}$. obtain

$$
\begin{aligned}
\gamma-\gamma_{r} \simeq & -\frac{\nu_{b a}}{2\left(\omega_{p b}^{2}+5 k^{2} v_{T b}^{2} / 3\right)}\left\{4 \omega_{p a}^{2}-\omega_{\mathrm{IA}}^{2}\right. \\
& \left.+\frac{2 k^{2} c_{s}^{2}}{\omega_{\mathrm{IA}}^{2}}\left[k^{2} v_{T a}^{2}\left(\frac{5}{3}+\frac{8 \nu_{a b}^{2}}{\omega_{\mathrm{IA}}^{2}}\right)+8 \nu_{a b}^{2}\right]\right\} .
\end{aligned}
$$

Hence, because always $\omega_{p a} \geqslant \omega_{\mathrm{IA}}$, here we have

$$
\gamma<\gamma_{r}
$$

i.e., the system relaxes on a time scale that is (much) shorter than the eventual growth time of the perturbations. Consequently, the assumed instability actually cannot develop. We note that this is in agreement with some experiments; e.g., in a Q-machine plasma ${ }^{15}$ where the instability has never been observed even by cooling the ions to near room temperature while keeping various temperatures for the electrons.

\section{SUMMARY}

To summarize, the long existing controversy dealing with the stability of the ion acoustic mode in plasmas in the state of partial thermodynamic equilibrium has been revisited. The results obtained here can be summarized as follows. (i) The friction does not affect the IA mode in the limit of quasineutral perturbations. (ii) Even using the nonevolving model equivalent to Ref. 4, there is no instability of the IA mode, contrary to claims from Ref. 4. (iii) When the background plasma is properly described as evolving in time, and as long as the quasineutrality is used, collisions do not produce a growth of the ion acoustic mode. (iv) When the Poisson equation is used instead of quasineutrality, in principle, there is a possibility for a positive growth rate of the IA mode. It appears as a combined effect of the breakdown of the charge neutrality from one side (introduced by the Poisson equation), and the heat transfer (the compressibility and advection in energy equation) from the other side, all within the background of a time-evolving plasma.

However, as the equilibrium plasma evolves in time, with the relaxation time $\tau_{r}$ given in Eq. (8), the obtained growth time must be (much) shorter than the relaxation time. Yet, this was shown to be impossible and we conclude that there is no instability in the electron-ion plasma with an initial temperature disparity if the plasma evolves freely. 


\section{ACKNOWLEDGMENTS}

Results were obtained in the framework of Projects Nos. G.0304.07 (FWO-Vlaanderen), C 90205 (Prodex), GOA/ 2004/01 (K.U. Leuven), and the Interuniversity Attraction Poles Programme-Belgian State-Belgian Science Policy. J.V. thanks N. D'Angelo for help and discussions.

${ }^{1}$ A. Okamoto, K. Hara, K. Nagaoka, S. Yoshimura, J. Vranjes, M. Kono, and M. Y. Tanaka, Phys. Plasmas 10, 2211 (2003).

${ }^{2}$ N. D’Angelo, Planet. Space Sci. 51, 393 (2003).

${ }^{3}$ R. L. Merlino and N. D'Angelo, Phys. Plasmas 12, 054504 (2005).

${ }^{4}$ T. D. Rognlien and S. A. Self, Phys. Rev. Lett. 27, 792 (1971).

${ }^{5}$ G. Bateman, Phys. Rev. Lett. 29, 1499 (1972).
${ }^{6}$ R. S. B. Ong and M. Y. Yu, Plasma Phys. 15, 659 (1973).

${ }^{7}$ R. J. Defouw, Phys. Rev. A 7, 818 (1973).

${ }^{8}$ J. E. Willet and H. Mehdian, J. Appl. Phys. 52, 6078 (1981).

${ }^{9}$ H. P. Wagner, H. J. Kaeppeler, and M. Auweter-Kurtz, J. Phys. D 31, 529 (1998).

${ }^{10}$ R. Sing and M. P. Bora, Phys. Plasmas 7, 2335 (2000).

${ }^{11}$ S. I. Braginskii, in Reviews of Plasma Physics (Consultants Bureau, New York, 1965), Vol. 1, p. 211.

${ }^{12}$ L. Landau, Zh. Eksp. Teor. Fiz. 7, 203 (1937).

${ }^{13}$ L. Spitzer, Physics of Fully Ionized Gasses (Interscience, New York, 1984), p. 135.

${ }^{14}$ J. Vranjes, D. Petrovic, B. P. Pandey, and S. Poedts, Phys. Plasmas 15, 072104 (2008).

${ }^{15}$ N. D'Angelo, private communication (2008). 